\title{
Adaptive fuzzy sliding-mode control for electrical servo drive
}

\author{
Rong-Jong Wai*, Chih-Min Lin, Chun-Fei Hsu \\ Department of Electrical Engineering, Yuan-Ze University, 135 Yuan-Tung Road, Chung-Li, Tao-Yuan 320, \\ Taiwan, ROC
}

Received 19 August 2002; received in revised form 8 April 2003; accepted 2 May 2003

\begin{abstract}
In this study, an adaptive fuzzy sliding-mode control (AFSMC) system with an integral-operation switching surface is adopted to control the position of an electrical servo drive. The AFSMC system is comprised of a fuzzy control design and a hitting control design. In the fuzzy control design a fuzzy controller is designed to mimic a feedback linearization (FL) control law. In the hitting control design a hitting controller is designed to compensate the approximation error between the FL control law and the fuzzy controller. The tuning algorithms are derived in the sense of the Lyapunov stability theorem, thus the stability of the system can be guaranteed. Moreover, to relax the requirement for the bound of approximation error, an error estimation mechanism is investigated to observe the bound of approximation error real-time. Experimental results verify that the proposed control systems can achieve favorable tracking performance and robust with regard to parameter variations and external load disturbance.
\end{abstract}

(C) 2003 Elsevier B.V. All rights reserved.

Keywords: Adaptive control; Fuzzy control; Sliding mode; Electrical servo drive

\section{Introduction}

Fuzzy control (FC) using linguistic information possesses several advantages such as robustness, model-free, universal approximation theorem and rule-based algorithm $[3,4,10]$. However, the huge amount of fuzzy rules for high-order systems makes the analysis complex. Recently, some researchers proposed fuzzy sliding-mode controllers (FSMC) $[2,8,15]$. Since only one variable (sliding surface) is defined as the fuzzy input variable, the main advantage of FSMC system is that the number of fuzzy rules is smaller than that for FLC which usually use the error and the change-of-error as the fuzzy input variables. Choi et al. used a single-input fuzzy variable called signed distance to design the fuzzy sliding-mode controller; however, the membership functions must be assumed to be isosceles

\footnotetext{
* Corresponding author. Tel.: +886-34638800 x429; fax: +886-34639355.

E-mail address: rjwai@saturn.yzu.edu.tw (R.-J. Wai).
} 
triangles with equal widths [2]. Palm proposed a sliding-mode fuzzy controller, which generates the absolute value of switching magnitude in the sliding-mode control law using the error and the changeof-error; however, too many fuzzy rules are required in the control process [8]. Yu et al. established a set of linear models to design the controller; however, their design method is not easy analytic for high-order systems [15]. Another researchers developed the adaptive fuzzy control (AFC) $[11,13,14]$. Based on the universal approximation theorem, the AFC design methods can provide stabilizing controller in the Lyapunov sense even for nonlinear systems with dominant uncertain nonlinearities by using sufficiently complex approximation functions [13]. With these approaches, the fuzzy rules can be automatically adjusted to achieve satisfactory system response by some dynamic adaptation laws. Since these control schemes use the error and the change-of-error as the fuzzy input variables, too many fuzzy rules are required in practical applications. Moreover, some strict constraints and prior knowledge of the controlled plant are necessary in the design process.

In recent years, the FC systems have been adopted to control electrical servo drives [5,12]. Liaw and Lin proposed a model following fuzzy adaptation mechanism to reduce the effects of parameter variations; however, the fuzzy rules must initially be constructed prior by a time-consuming trialand-error tuning procedure [5]. Tzou and Lin proposed a fuzzy-tuning current-vector control scheme; however, the stability of the system cannot be guaranteed [12]. On the other hand, the combination of adaptive technique and intelligent control (fuzzy control or neural network control) for electrical servo drives has also grown rapidly [6,7]. Lin and Chiu developed an adaptation law to obtain the upper bound of uncertainties for an adaptive fuzzy sliding-mode control system; however, when the system parameters have large variations, the serious chattering phenomena will results in the control effort [6]. Lin et al. combined a linear model-following controller and an on-line trained neural network to compensate for the uncertainties of an induction servo motor drive; however, the design procedure is overtly complex [7].

The motivation of this study is to design an adaptive fuzzy sliding-mode control (AFSMC) scheme to overcome the mentioned drawbacks in preview works. The AFSMC possesses the advantages that it can automatically adjust the fuzzy rules like the AFC and can reduce the fuzzy rules like FSMC. The developed AFSMC system tries to address those problems: (1) assume no mathematical model of the system; (2) can incorporate linguistic information from human experts directly into the controllers; and (3) guarantee stability of the resulting closed-loop system in the sense of Lyapunov stability theorem. Moreover, to relax the requirement for the bound of approximation error, a simple estimation algorithm is investigated to observe the bound of approximation error real-time. According to the on-line adjustment, the chattering control effort can be much reduced. In addition, experimental results are provided to verify the effectiveness of the AFSMC systems.

\section{Electrical servo drive}

In general, the mechanical equation of an electrical servo drive (e.g. direct-current motor drive, induction motor drive or permanent magnet synchronous motor drive) can be represented as [1]

$$
J \ddot{\theta}(t)+B \dot{\theta}(t)+T_{1}=T_{\mathrm{e}},
$$

where $J$ is the moment of inertia; $B$ is the damping coefficient; $\theta$ is the rotor position; $T_{1}$ represents the external load disturbance, nonlinear friction and unpredicted uncertainties; $T_{\mathrm{e}}$ denotes the electric 
torque. With suitable impressed current or field oriented control $[1,5]$, the electromagnetic torque can be simplified as

$$
T_{\mathrm{e}}=K_{\mathrm{t}} i,
$$

where $K_{\mathrm{t}}$ is the torque constant and $i$ is the control current. Substituting (2) into (1), then the electrical servo drive system can be rewritten in the following form:

$$
\ddot{\theta}(t)=-\frac{B}{J} \dot{\theta}(t)+\frac{K_{\mathrm{t}}}{J} i(t)-\frac{1}{J} T_{1} \equiv A_{p} \dot{\theta}(t)+B_{p} u(t)+D_{p} T_{1},
$$

where $A_{p}=-B / J, B_{p}=K_{\mathrm{t}} / J>0, D_{p}=-1 / J$ and $u(t)=i(t)$ is the control effort.

\section{Fuzzy sliding-mode control}

The block diagram of a FSMC system for electrical servo drive is depicted in Fig. 1, in which $\theta_{\mathrm{c}}(t)$ is the tracking command. The control problem is to find a suitable control law so that the rotor position can track specific reference trajectories. Define a tracking error as

$$
e(t)=\theta(t)-\theta_{\mathrm{c}}(t) .
$$

The first step of sliding-mode control design is to select a sliding surface that models the desired closed-loop performance in state variable space. Then design the control such that the system state trajectories are forced toward the sliding surface and stay on it. Now, suppose that an integraloperation sliding surface is given as

$$
s(t)=\dot{\theta}(t)-\int_{0}^{t}\left[\ddot{\theta}_{\mathrm{c}}(\tau)-k_{1} \dot{e}(\tau)-k_{2} e(\tau)\right] \mathrm{d} \tau,
$$

where $k_{1}$ and $k_{2}$ are non-zero positive constants. From (5), if the state trajectory of system (3) is trapped on the sliding surface, namely $s(t)=\dot{s}(t)=0$, then the equivalent dynamics of system (3) is

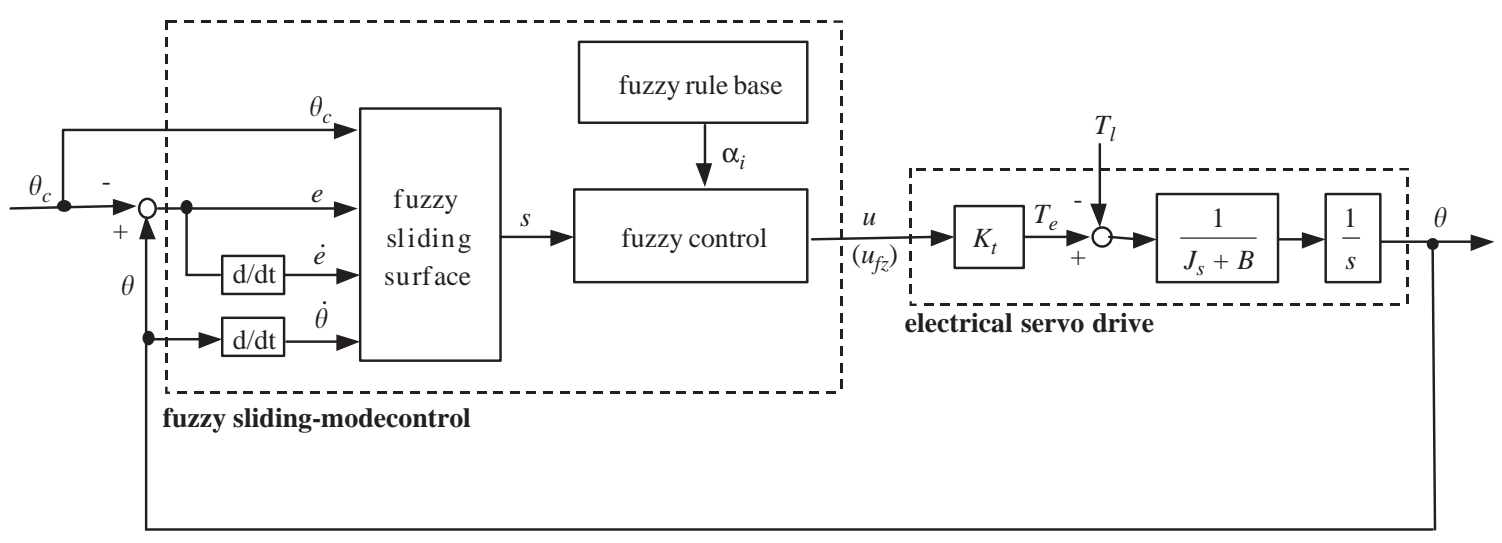

Fig. 1. Fuzzy sliding-mode control for electrical servo drive. 


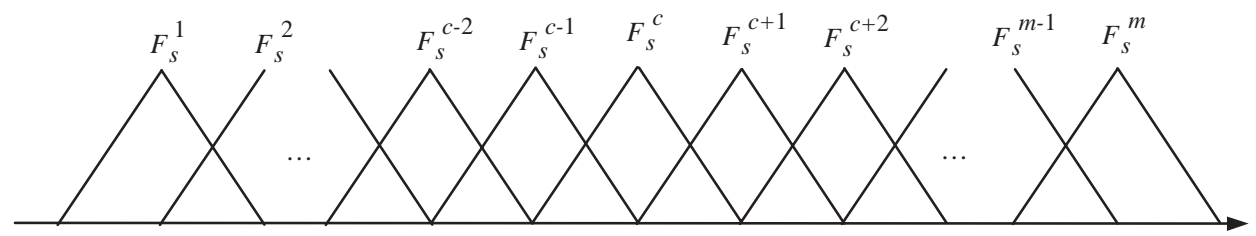

(a)

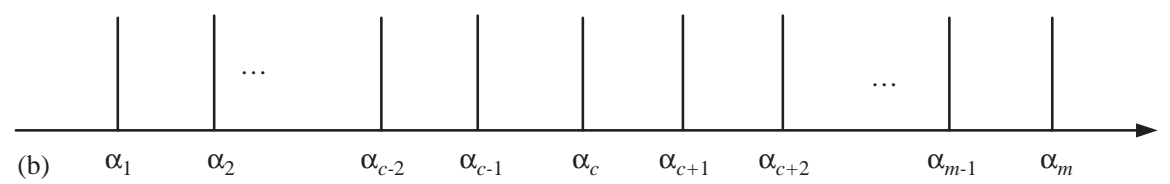

Fig. 2. (a) Membership function of IF-part; (b) membership function of THEN-part.

governed by

$$
\ddot{e}(t)+k_{1} \dot{e}(t)+k_{2} e(t)=0 .
$$

It is obvious that the tracking error $e(t)$ will converge to zero exponentially if the gains, $k_{1}$ and $k_{2}$, are selected properly.

The complete rule base of a conventional fuzzy system with $n$ input variables has $p^{n}$ rules, where $p$ is the number of linguistic terms per input variable. As the dimension and complexity of a system increase, the size of the rule base increases exponentially. By defining the sliding surface as the input variable of fuzzy rules, the number of fuzzy rules for FSMC is smaller than that for FC, which usually uses the error and the change-of-error as the input variables. The fuzzy rules are given in the following form [2]:

Rule $i$ : IF $s$ is $F_{s}^{i}$, THEN $u$ is $\alpha_{i}$,

where $\alpha_{i}, i=1,2, \ldots, m$ are the singleton control actions and $F_{s}^{i}$ is the label of the fuzzy set. The triangular-typed functions and singletons are used to define the membership functions of IF-part and THEN-part, which are depicted in Figs. 2(a) and (b), respectively. The defuzzification of the control output is accomplished by the method of center-of-gravity [4]

$$
u_{\mathrm{fz}}(s)=\frac{\sum_{i=1}^{m} w_{i} \times \alpha_{i}}{\sum_{i=1}^{m} w_{i}},
$$

where $w_{i}$ is the firing weight of the $i$ th rule.

\section{Adaptive fuzzy sliding-mode control}

Assume that the system dynamics are well known and the external load disturbance is measurable, a feedback linearization (FL) control law that achieves $\dot{s}(t)=0$ can be obtained from (3) and (5)

$$
u^{*}(t)=B_{p}^{-1}\left[-A_{p} \dot{\theta}(t)-D_{p} T_{1}+\ddot{\theta}_{\mathrm{c}}(t)-k_{1} \dot{e}(t)-k_{2} e(t)\right] .
$$


Substituting (9) into (3) gives

$$
\ddot{e}(t)+k_{1} \dot{e}(t)+k_{2} e(t)=0 .
$$

If the control gains $k_{1}$ and $k_{2}$ are properly chosen such that the characteristic polynomial of (10) is strictly Hurwitz, that is a polynomial whose roots lie strictly in the open left half of the complex plane, it implies that $\lim _{t \rightarrow \infty} e(t)=0$ meaning that the closed-loop system is globally asymptotically stable. Since the system dynamics and the external load disturbance may be unknown or perturbed, the FL control law $u^{*}(t)$ cannot be implemented in practical applications. Therefore, an AFSMC system is proposed to mimic the FL control law in this study. If $\alpha_{i}$ is chosen as an adjustable parameter, (8) can be rewritten as

$$
u_{\mathrm{fz}}(s, \boldsymbol{\alpha})=\boldsymbol{\alpha}^{\mathrm{T}} \boldsymbol{\xi}
$$

where $\boldsymbol{\alpha}=\left[\alpha_{1}, \alpha_{2}, \ldots, \alpha_{m}\right]^{\mathrm{T}}$ is a parameter vector and $\xi=\left[\xi_{1}, \xi_{2}, \ldots, \xi_{m}\right]^{\mathrm{T}}$ is a regressive vector with $\xi_{i}$ defined as

$$
\xi_{i}=\frac{w_{i}}{\sum_{i=1}^{m} w_{i}}
$$

According to the universal approximation theorem [13], there exists an optimal fuzzy control system $u_{\mathrm{fz}}^{*}\left(s, \boldsymbol{\alpha}^{*}\right)$ in the form of (11) such that

$$
u^{*}(t)=u_{\mathrm{fz}}^{*}\left(s, \boldsymbol{\alpha}^{*}\right)+\varepsilon=\boldsymbol{\alpha}^{* \mathrm{~T}} \xi+\varepsilon,
$$

where $\varepsilon$ is the approximation error and is assumed to be bounded by $|\varepsilon|<E$. Employing a fuzzy control system $\hat{u}_{\mathrm{fz}}(s, \hat{\boldsymbol{\alpha}})$ to approximate $u^{*}(t)$

$$
\hat{u}_{\mathrm{fz}}(s, \hat{\alpha})=\hat{\alpha}^{\mathrm{T}} \xi
$$

where $\hat{\alpha}$ is the estimated vector of $\alpha^{*}$.

The block diagram of an AFSMC system for electrical servo drive is depicted in Fig. 3. The control law for the developed AFSMC is assumed to take the following form:

$$
u(t)=\hat{u}_{\mathrm{fz}}(s, \hat{\alpha})+u_{\mathrm{vs}}(s),
$$

where the fuzzy control $\hat{u}_{\mathrm{fz}}$ is the main tracking controller to mimic the FL control law $u^{*}(t)$ and the hitting control $u_{\mathrm{vs}}$ is designed to compensate the difference between the FL control law and the fuzzy controller. By substituting (15) into (3), it is revealed that

$$
\ddot{\theta}(t)=A_{p} \dot{\theta}(t)+B_{p}\left[\hat{u}_{\mathrm{fz}}+u_{\mathrm{vs}}\right]+D_{p} T_{1} .
$$

After some straightforward manipulation, the error equation governing the closed-loop system can be obtained through (5), (9) and (16) as follows:

$$
\ddot{e}(t)+k_{1} \dot{e}(t)+k_{2} e(t)=B_{p}\left[\hat{u}_{\mathrm{fz}}+u_{\mathrm{vs}}-u^{*}\right]=\dot{s}(t) .
$$




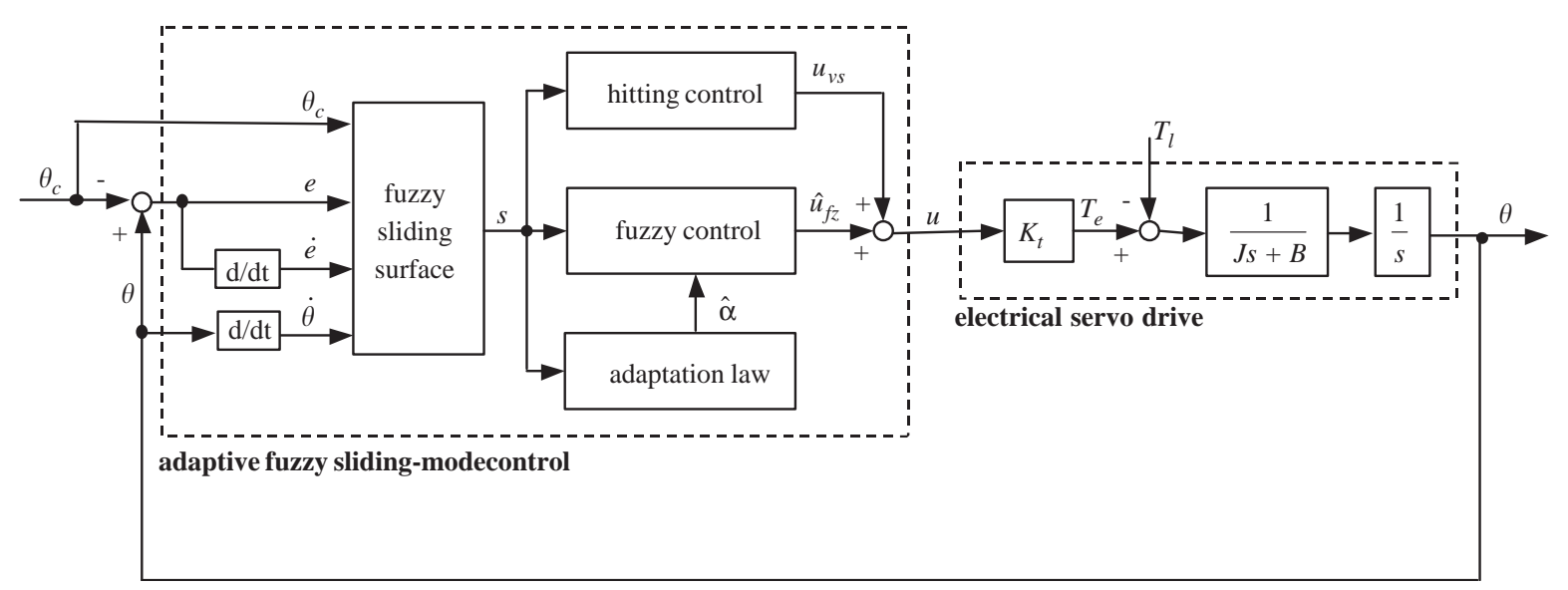

Fig. 3. Adaptive fuzzy sliding-mode control for electrical servo drive.

And, $\tilde{u}_{\mathrm{fz}}$ is denoted as

$$
\tilde{u}_{\mathrm{fz}} \equiv \hat{u}_{\mathrm{fz}}-u^{*}=\hat{u}_{\mathrm{fz}}-u_{\mathrm{fz}}^{*}-\varepsilon .
$$

For simplicity of discussion, define $\tilde{\alpha}=\hat{\alpha}-\alpha^{*}$ to obtain a rewritten form of (18) via (13) and (14) as

$$
\tilde{u}_{\mathrm{fz}}=\tilde{\alpha}^{\mathrm{T}} \xi-\varepsilon .
$$

The basic philosophy of Lyapunov's method is the mathematical extension of a fundamental physical observation: if the total energy of a system is continuously dissipated, then the system must eventually settle down to equilibrium states. Thus, it may conclude the stability of a system by examining the descent variation of an energy function (Lyapunov function) for introducing a suitable control law and associated adaptation rules. In order to force the states $s(t)$ and $\tilde{\boldsymbol{\alpha}}$ tend to zero, consider a Lyapunov function candidate in the following form:

$$
V_{1}(s(t), \tilde{\boldsymbol{\alpha}})=\frac{1}{2} s^{2}(t)+\frac{B_{p}}{2 \eta_{1}} \tilde{\boldsymbol{\alpha}}^{\mathrm{T}} \tilde{\boldsymbol{\alpha}},
$$

where $\eta_{1}$ is a positive constant. Differentiating (20) with respect to time, it can obtain that

$$
\begin{aligned}
\dot{V}_{1}(s(t), \tilde{\boldsymbol{\alpha}}) & =s(t) \dot{s}(t)+\frac{B_{p}}{\eta_{1}} \tilde{\boldsymbol{\alpha}}^{\mathrm{T}} \dot{\tilde{\boldsymbol{\alpha}}}=s(t) B_{p}\left(\hat{u}_{\mathrm{fz}}+u_{\mathrm{vs}}-u^{*}\right)+\frac{B_{p}}{\eta_{1}} \tilde{\boldsymbol{\alpha}}^{\mathrm{T}} \dot{\tilde{\alpha}} \\
& =s(t) B_{p}\left(\tilde{\boldsymbol{\alpha}}^{\mathrm{T}} \xi+u_{\mathrm{vs}}-\varepsilon\right)+\frac{B_{p}}{\eta_{1}} \tilde{\boldsymbol{\alpha}}^{\mathrm{T}} \dot{\tilde{\boldsymbol{\alpha}}}=B_{p} \tilde{\boldsymbol{\alpha}}^{\mathrm{T}}\left(s(t) \xi+\frac{1}{\eta_{1}} \dot{\tilde{\boldsymbol{\alpha}}}\right)+s(t) B_{p}\left(u_{\mathrm{vs}}-\varepsilon\right) .
\end{aligned}
$$

For achieving $\dot{V}_{1} \leqslant 0$, the adaptation law and hitting controller are designed as

$$
\begin{aligned}
& \dot{\tilde{\alpha}}=\dot{\hat{\alpha}}=-\eta_{1} s(t) \xi, \\
& u_{\mathrm{vs}}=-E \operatorname{sgn}(s(t)),
\end{aligned}
$$


where $\operatorname{sgn}(\cdot)$ is a sign function. Then $(21)$ can be rewritten as

$$
\begin{aligned}
\dot{V}_{1}(s(t), \tilde{\boldsymbol{\alpha}}) & =-E|s(t)| B_{p}-\varepsilon s(t) B_{p} \leqslant-E|s(t)| B_{p}+|\varepsilon||s(t)| B_{p} \\
& =-(E-|\varepsilon|)|s(t)| B_{p} \leqslant 0
\end{aligned}
$$

This implies that $\dot{V}_{1}$ is a negative semi-definite function. Define the following term

$$
P(t) \equiv(E-|\varepsilon|)|s(t)| B_{p} \leqslant-\dot{V}_{1}(s(t), \tilde{\alpha}) .
$$

Because $V_{1}(s(0), \tilde{\alpha})$ is bounded and $V_{1}(s(t), \tilde{\alpha})$ is non-increasing and bounded, then

$$
\int_{0}^{t} P(\tau) \mathrm{d} \tau \leqslant V_{1}(s(0), \tilde{\boldsymbol{\alpha}})-V_{1}(s(t), \tilde{\boldsymbol{\alpha}})<\infty .
$$

Also, $\dot{P}(t)$ is bounded, it can be shown that $\lim _{t \rightarrow \infty} P(t)=0$ by Barbalat's Lemma [9]. That is, $s(t) \rightarrow 0$ as $t \rightarrow \infty$. In summary, the AFSMC system is presented in (15), where $\hat{u}_{\mathrm{fz}}$ is given in (14) with the parameters $\hat{\boldsymbol{\alpha}}$ adjusted by (22) and $u_{\mathrm{vs}}$ is given in (23). By applying this adaptive law and hitting control law, the AFSMC system can be guaranteed to be stable in the Lyapunov sense.

\section{Adaptive fuzzy sliding-mode control with bound estimation}

In Section 4, the application of the AFSMC system requires the bound of approximation error. However, the bound of approximation error $E$ is difficult to measure for practical applications in industry. If $E$ is chosen too large, the control effort results large chattering. The chattering phenomenon in the control effort will wear the bearing mechanism and excite unstable dynamics. If $E$ is chosen too small, the control system may be unstable. For an application in practical design, the bound of approximation error is chosen large enough to avoid unstable. To relax the requirement for the bound of approximation error, the AFSMC system with bound estimation for electrical servo drive is depicted in Fig. 4. Replacing $E$ by $\hat{E}(t)$ in (23), the following equation can be obtained:

$$
u_{\mathrm{vs}}=-\hat{E}(t) \operatorname{sgn}(s(t))
$$

where $\hat{E}(t)$ is the estimated bound value of the approximation error. Define an estimated error as

$$
\tilde{E}(t)=\hat{E}(t)-E .
$$

In order to force the states $s(t), \tilde{\alpha}$ and $\tilde{E}$ tend to zero, define a Lyapunov function candidate as

$$
V_{2}(s(t), \tilde{\boldsymbol{\alpha}}, \tilde{E})=\frac{1}{2} s^{2}(t)+\frac{B_{p}}{2 \eta_{1}} \tilde{\boldsymbol{\alpha}}^{\mathrm{T}} \tilde{\boldsymbol{\alpha}}+\frac{B_{p}}{2 \eta_{2}} \tilde{E}^{2},
$$

where $\eta_{2}$ is a positive constant. Differentiating (29) with respect to time and using (22) and (27), it can obtain that

$$
\dot{V}_{2}(s(t), \tilde{\boldsymbol{\alpha}}, \tilde{E})=s(t) \dot{s}(t)+\frac{B_{p}}{\eta_{1}} \tilde{\boldsymbol{\alpha}}^{\mathrm{T}} \dot{\tilde{\boldsymbol{\alpha}}}+\frac{B_{p}}{\eta_{2}} \tilde{E} \dot{\tilde{E}}
$$




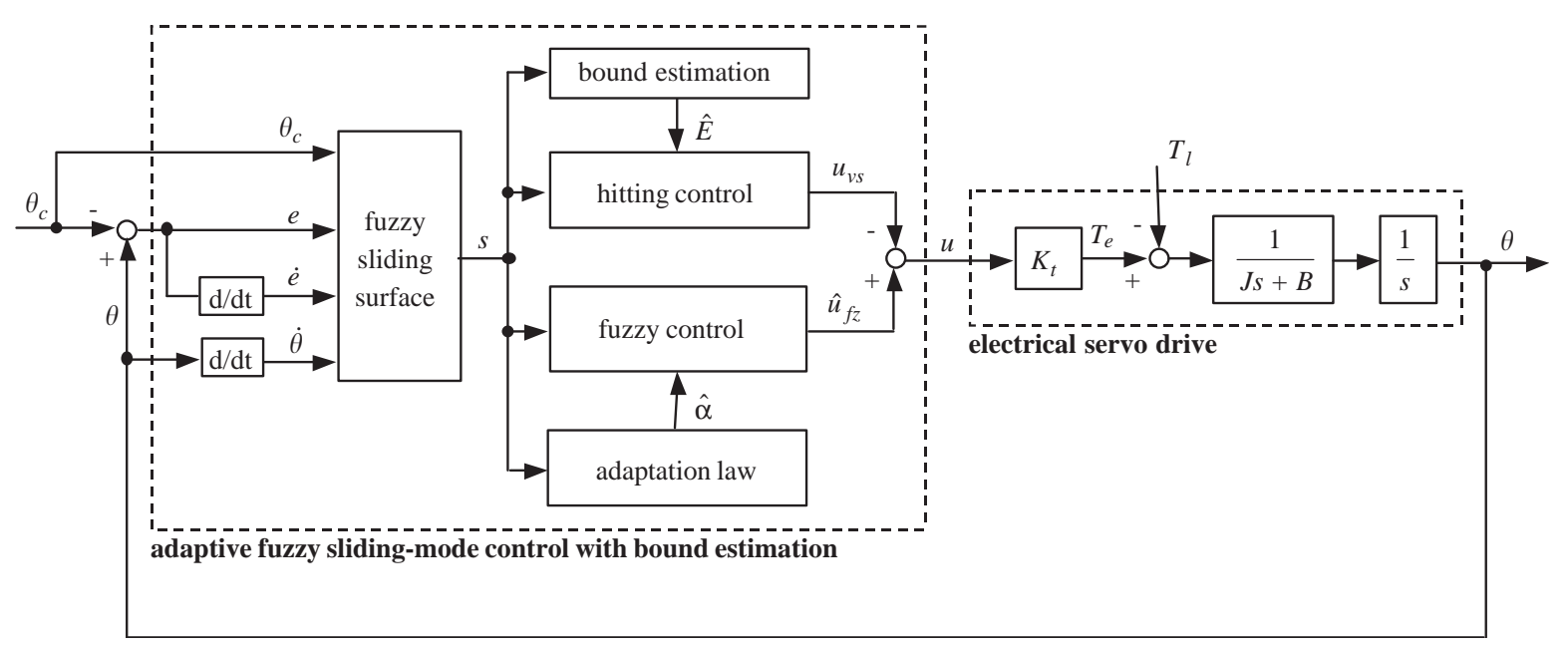

Fig. 4. Adaptive fuzzy sliding-mode control with bound estimation for electrical servo drive.

$$
\begin{aligned}
& =B_{p} \tilde{\boldsymbol{\alpha}}^{\mathrm{T}}\left(s(t) \xi+\frac{1}{\eta_{1}} \dot{\hat{\boldsymbol{\alpha}}}\right)+s(t) B_{p}\left(u_{\mathrm{vs}}-\varepsilon\right)+\frac{B_{p}}{\eta_{2}} \tilde{E} \dot{\tilde{E}} \\
& =-\hat{E}(t)|s(t)| B_{p}-\varepsilon s(t) B_{p}+\frac{B_{p}}{\eta_{2}}[\hat{E}(t)-E] \dot{\hat{E}}(t) .
\end{aligned}
$$

For achieving $\dot{V}_{2} \leqslant 0$, the estimation law is designed as

$$
\dot{\hat{E}}(t)=\eta_{2}|s(t)|,
$$

then (30) can be rewritten as

$$
\begin{aligned}
\dot{V}_{2}(s(t), \tilde{\alpha}, \tilde{E}) & =-\hat{E}|s(t)| B_{p}-\varepsilon s(t) B_{p}+(\hat{E}-E)|s(t)| B_{p} \\
& =-\varepsilon s(t) B_{p}-E|s(t)| B_{p} \leqslant|\varepsilon||s(t)| B_{p}-E|s(t)| B_{p}=-(E-|\varepsilon|)|s(t)| B_{p} \leqslant 0 .
\end{aligned}
$$

By Barbalat's lemma [9], it can conclude that $s(t) \rightarrow 0$ as $t \rightarrow \infty$. In summary, the AFSMC system with bound estimation is presented in (15), where $\hat{u}_{\mathrm{fz}}$ is given in (14) with the parameters $\hat{\alpha}$ adjusted by (22); $u_{\mathrm{vs}}$ is given in (27) with the parameter $\hat{E}$ adjusted by (31). By applying this estimation law, the AFSMC system with bound estimation can be guaranteed to be stable in the Lyapunov sense.

\section{Experimental results}

The electrical servo drive used in this study is a three-phase Y-connected four-pole $800 \mathrm{~W} 60 \mathrm{~Hz}$ $120 \mathrm{~V} / 5.4 \mathrm{~A}$ type induction servomotor. For the position control system, the braking machine is driven by a current source drive to provide the braking torque (external load disturbance). A servo 
Table 1

Fuzzy rules of FSMC system for electrical servo drive

\begin{tabular}{|c|c|c|c|c|c|c|c|}
\hline$s$ & NB & $\mathrm{NM}$ & NS & $\mathrm{ZO}$ & PS & $\mathrm{PM}$ & PB \\
\hline$\alpha$ & 5.00 & 3.00 & 1.00 & 0.00 & -1.00 & -3.00 & -5.00 \\
\hline
\end{tabular}

control card is installed in a control computer, which includes multi-channels of D/A and encoder interface circuits. Digital filter and frequency multiplied by four circuits are built into the encoder interface circuit to increase the precision of position feedback. The proposed control systems are realized in a Pentium CPU via "Turbo C" language and the control interval of the position control loop is set at $2 \mathrm{~ms}$. It is worth to mention that the limitation of the control effort is $\pm 10 \mathrm{~A}$ according to the D/A resolution of the servo control card and the electrical specification of the induction motor in real drive system. This limitation may discount the trajectory tracking quality of the proposed control systems, and it can be solved via a servo control card with high resolution or the selection of the control parameters carefully. Two examined conditions are given to verify the robustness of the proposed control schemes. One is the external disturbance condition, that is the nominal inertia with $1 \mathrm{~N} \mathrm{~m}$ braking-load disturbance occurring at $4.5 \mathrm{~s}$, and the other is the parameter variation condition, that is the increasing of the rotor inertia to approximately three times the nominal value with $1 \mathrm{~N} \mathrm{~m}$ braking-load disturbance occurring at $4.5 \mathrm{~s}$.

The parameters of the proposed control systems are selected as follows:

$$
k_{1}=10, k_{2}=25, \eta_{1}=200, \eta_{2}=0.5, E=1
$$

Properly choosing the values of $k_{1}$ and $k_{2}$, the desired system dynamics such as rise time, overshoot, and settling time can be easily designed by the second-order system shown in (10). Moreover, the gains, $\eta_{1}$ and $\eta_{2}$, in the proposed control systems are chosen to achieve the superior transient responses by trial and error in the experimentation considering the requirement of stability and the limitation of control effort. Note that, introducing $\eta_{1}$ and $\eta_{2}$ into the derivation process can tune the convergent speed of the adaptation and estimation laws, and it also can be ignored in (20) and (29). In addition, the fixed bound $E$ in the AFSMC system can be determined roughly according to the possible operating conditions. Note that, the bound value increases as the amplitude or the frequency of reference trajectory raises since it is concerned with the kind of reference trajectory.

First, a FSMC with fixed fuzzy rules is adopted to control the electrical servo drive for comparison. The associated fuzzy rules are summarized in Table 1, in which the fuzzy labels used in this study are negative big $(\mathrm{NB})$, negative medium $(\mathrm{NM})$, negative small $(\mathrm{NS})$, zero $(\mathrm{ZO})$, positive small (PS), positive medium (PM) and positive big (PB). The position responses, control efforts and tracking errors of the FSMC system for periodic sinusoidal and triangular commands at examined conditions are depicted in Figs. 5 and 6, respectively. Although favorable tracking responses can be obtained at nominal condition; however, the fuzzy rules must be tuned by time-consuming trial-anderror procedure and the stability of the control system cannot be guaranteed at all times. Besides, when the external disturbance and parameter variations occur, the degenerate tracking responses are resulted. To solve these problems, an AFSMC system for electrical servo drive is developed. With 


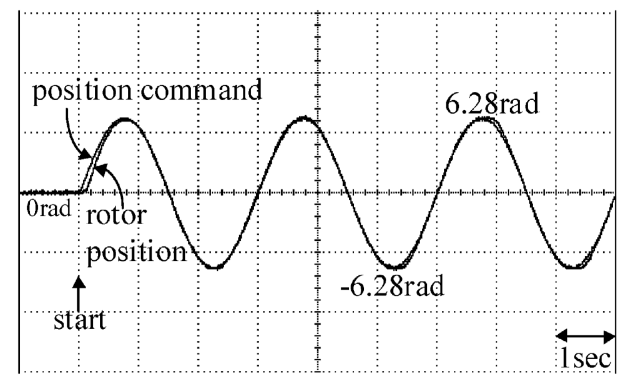

(a)

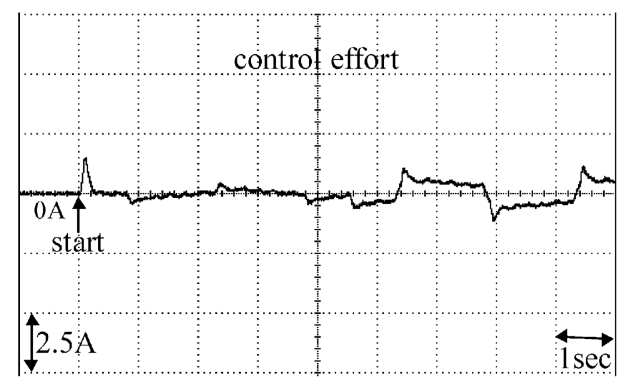

(b)

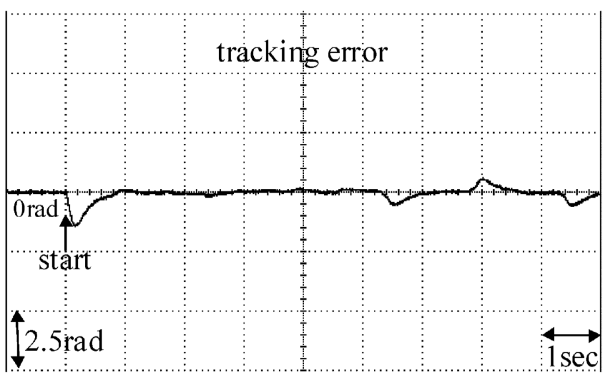

(c)

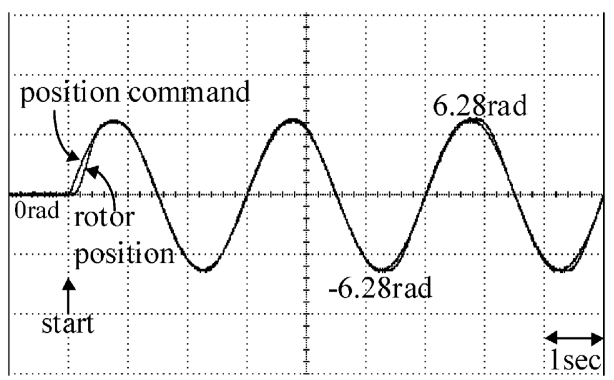

(d)

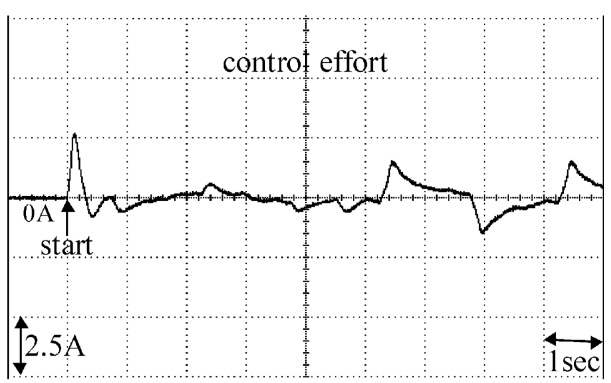

(e)

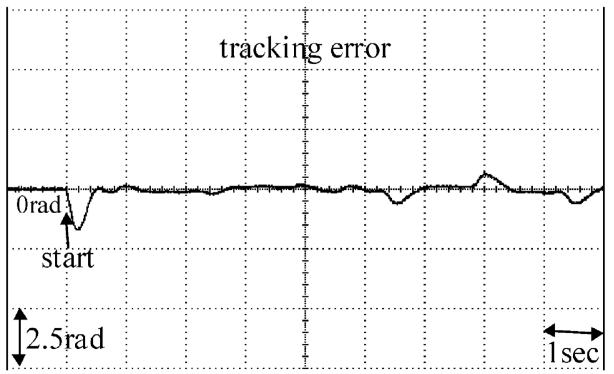

(f)

Fig. 5. Experimental results of FSMC system for periodic sinusoidal command: (a) position response at external disturbance condition; (b) control effort at external disturbance condition; (c) tracking error at external disturbance condition; (d) position response at parameter variation condition; (e) control effort at parameter variation condition; (f) tracking error at parameter variation condition.

this approach, the fuzzy rules can be automatically adjusted to achieve satisfactory system response by adaptation laws and the stability of the system can be guaranteed in the Lyapunov sense. The position responses, control efforts and tracking errors of the AFSMC system for periodic sinusoidal and triangular commands at examined conditions are depicted in Figs. 7 and 8, respectively. The robust control performance of the AFSMC system is obvious under the occurrence of the parameter variations and external load disturbance. However, the undesirable chattering phenomena in the control efforts, which are depicted in Figs. 7(b), (e), 8(b) and (e), are serious due to the excess selection of a bound value in the hitting controller (23). The undesired chattering control efforts will wear the bearing mechanism and might excite unstable system dynamics. Now, an 


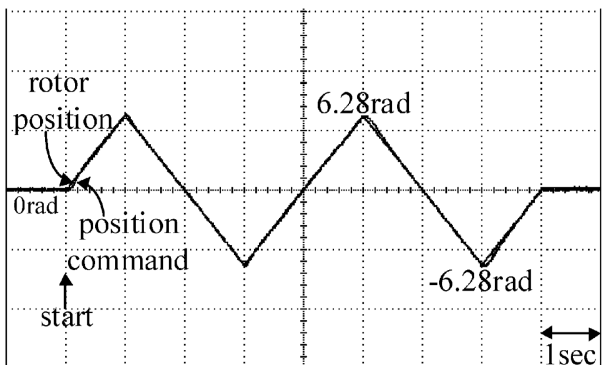

(a)

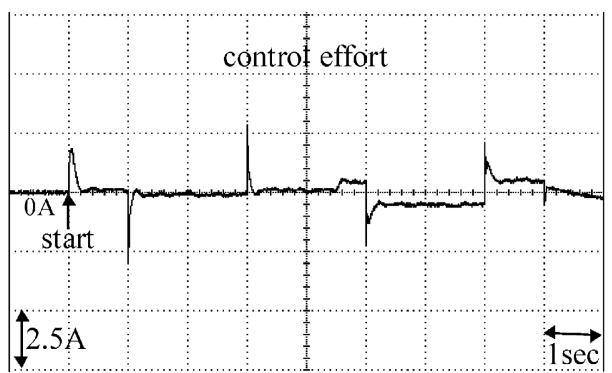

(b)

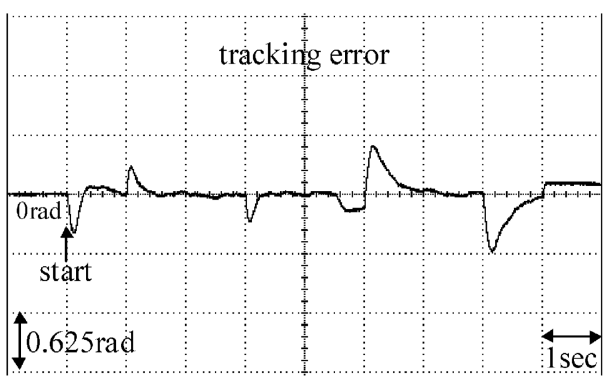

(c)

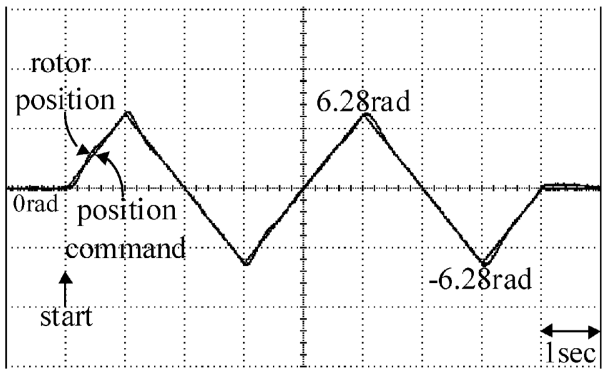

(d)

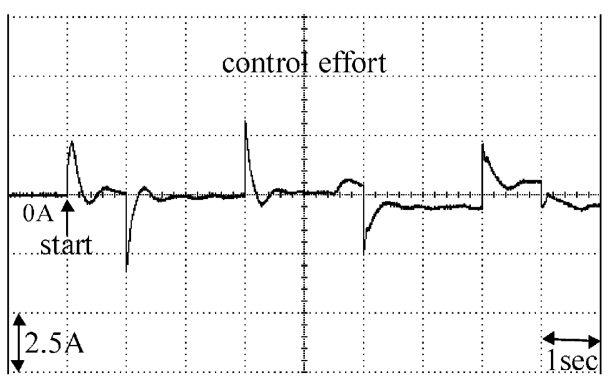

(e)

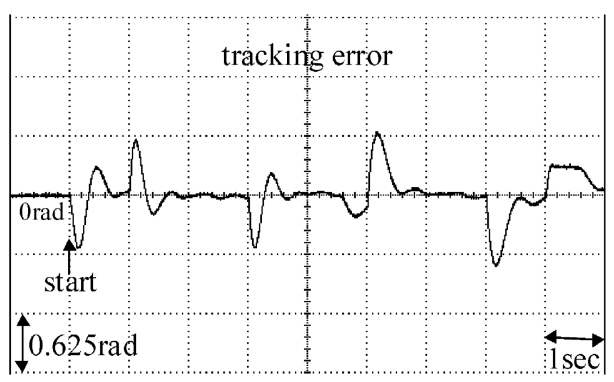

(f)

Fig. 6. Experimental results of FSMC system for periodic triangular command: (a) position response at external disturbance condition; (b) control effort at external disturbance condition; (c) tracking error at external disturbance condition; (d) position response at parameter variation condition; (e) control effort at parameter variation condition; (f) tracking error at parameter variation condition.

AFSMC system with bound estimation is applied to control the electrical servo drive. The position responses, control efforts and tracking errors of the AFSMC system with bound estimation for periodic sinusoidal and triangular commands at examined conditions are depicted in Figs. 9 and 10, respectively. From the experimental results, the robust control performance also can be obtained; moreover, the chattering phenomena are much reduced in the control efforts according to the on-line adjustment of the bound value in the hitting controller. Compared the experimental results shown in Figs. 5-10, the proposed AFSMC system with bound estimation is more suitable to control the rotor position of the electrical servo drive under the possible occurrence of uncertainties. 


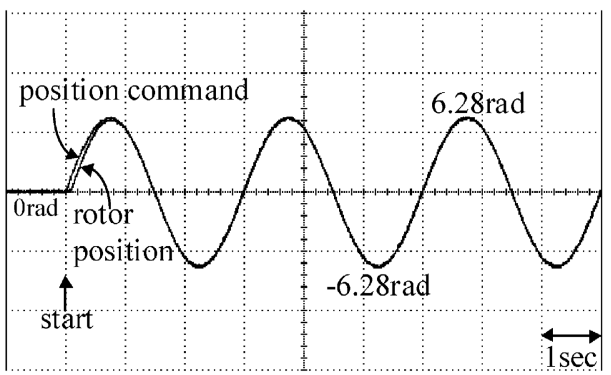

(a)

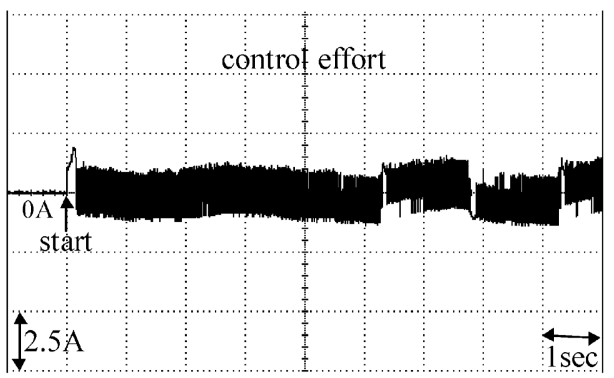

(b)

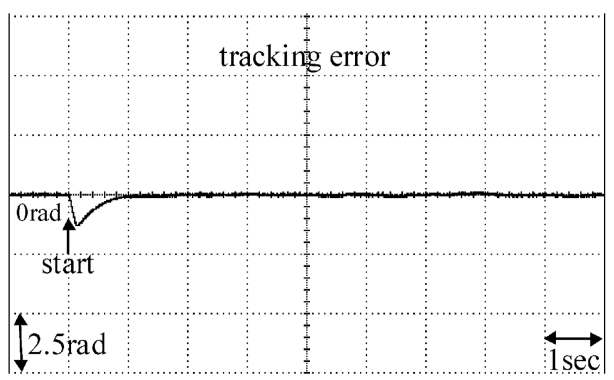

(c)

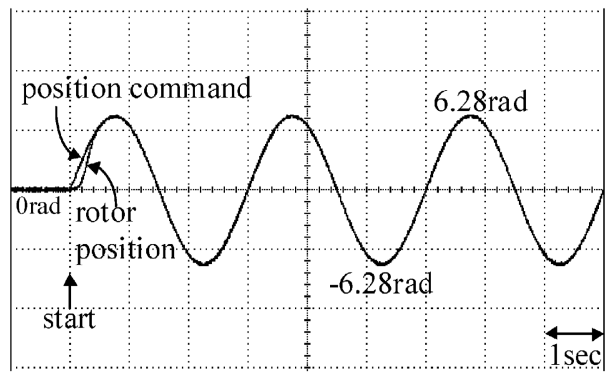

(d)

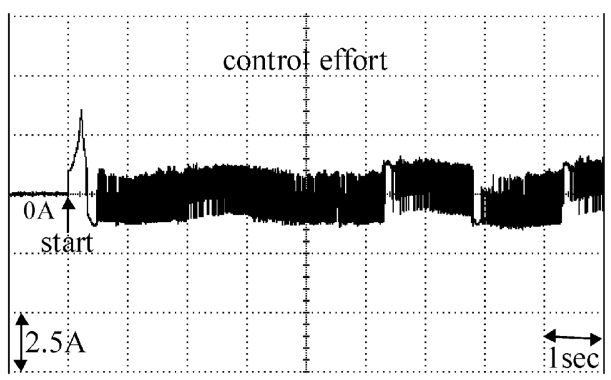

(e)

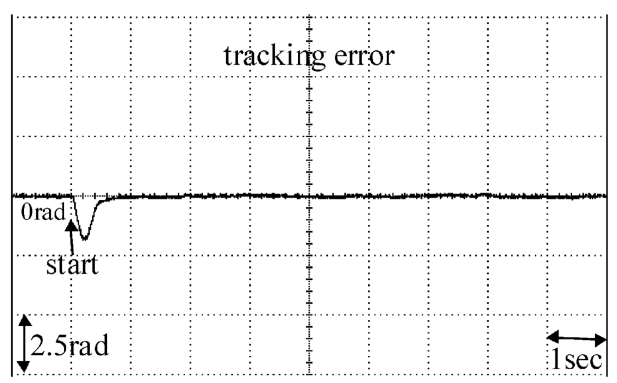

(f)

Fig. 7. Experimental results of AFSMC system for periodic sinusoidal command: (a) position response at external disturbance condition; (b) control effort at external disturbance condition; (c) tracking error at external disturbance condition; (d) position response at parameter variation condition; (e) control effort at parameter variation condition; (f) tracking error at parameter variation condition.

The conventional non-adaptive fuzzy control scheme is easy to perform in industry due to their simple control structure, ease of design, and inexpensive cost. However, the FSMC with fixed fuzzy rules cannot provide perfect control performance if the controlled plant is highly nonlinear and uncertain. Though the AFSMC system with bound estimation expenses extra time with more memory bank to execute the adaptation and estimation laws, it results in superior control performance than the FSMC system. Moreover, recent development in microelectronics and very large scale integration (VLSI) has pushed the performance of microprocessors to an unprecedented level with ever lower-cost. Today high-performance microprocessors and digital signal processor (DSP) can be effectively used to provide flexible environments with high execution rate for advanced control schemes. 


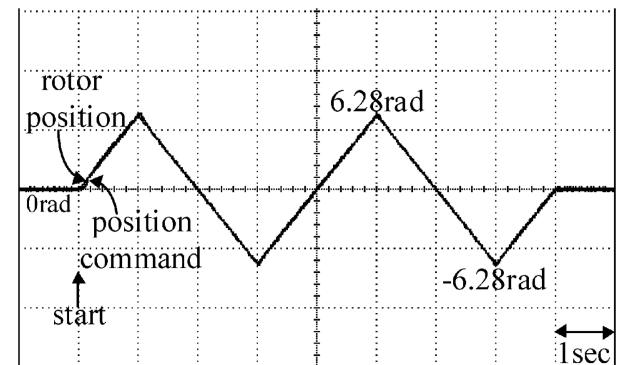

(a)

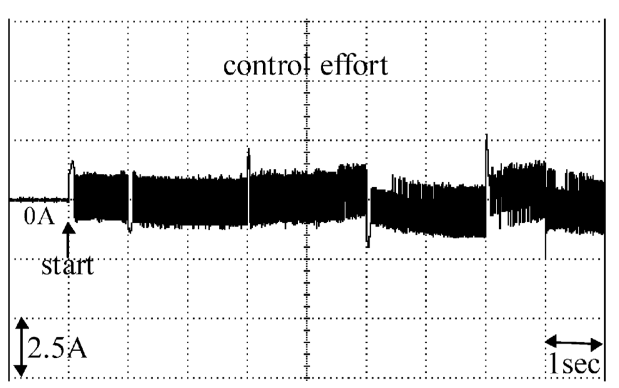

(b)

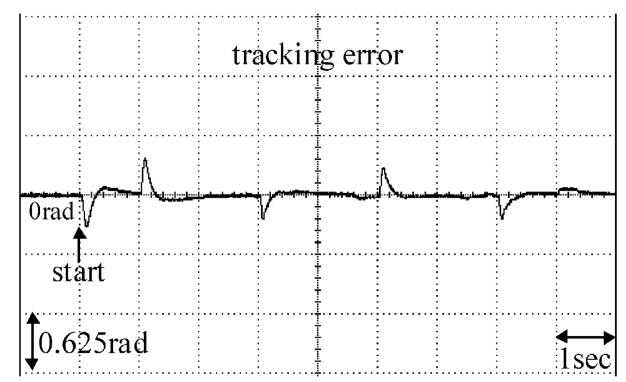

(c)

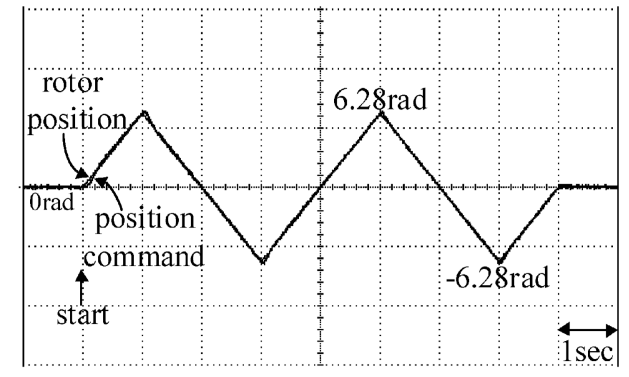

(d)

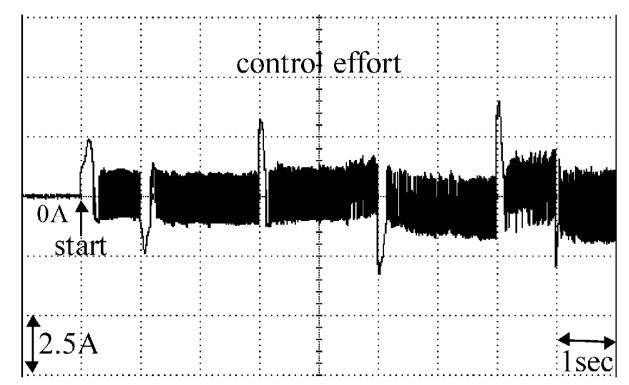

(e)

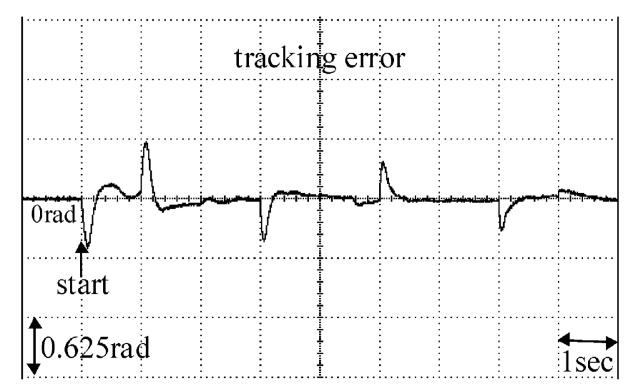

(f)

Fig. 8. Experimental results of AFSMC system for periodic triangular command: (a) position response at external disturbance condition; (b) control effort at external disturbance condition; (c) tracking error at external disturbance condition; (d) position response at parameter variation condition; (e) control effort at parameter variation condition; (f) tracking error at parameter variation condition.

\section{Conclusions}

In this study, a fuzzy sliding-mode control (FSMC), an adaptive fuzzy sliding-mode control (AFSMC) and an AFSMC with bound estimation have been adopted to control the rotor position of a computer-controlled electrical servo drive. This study has successfully demonstrated the adaptive technique applied to the design of the stable fuzzy controller. The adaptation laws based on the Lyapunov stability theorem can automatically adjust the fuzzy rules. Thus, the stability of the developed AFSMC system can be guaranteed. Moreover, to relax the requirement for the bound value in the hitting control, an AFSMC system with bound estimation was investigated to control the electrical servo drive. According to the experimental results of the AFSMC system 


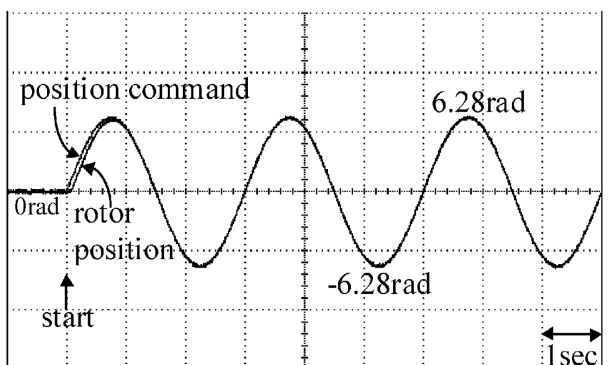

(a)

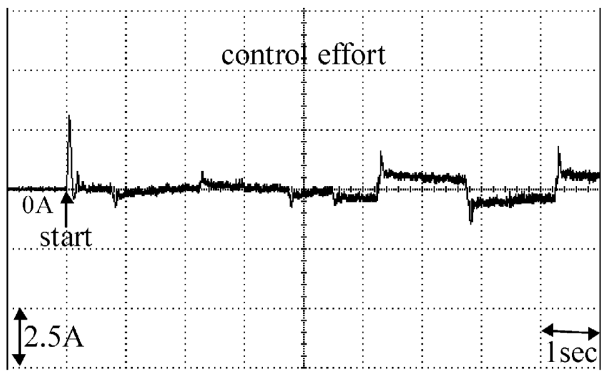

(b)

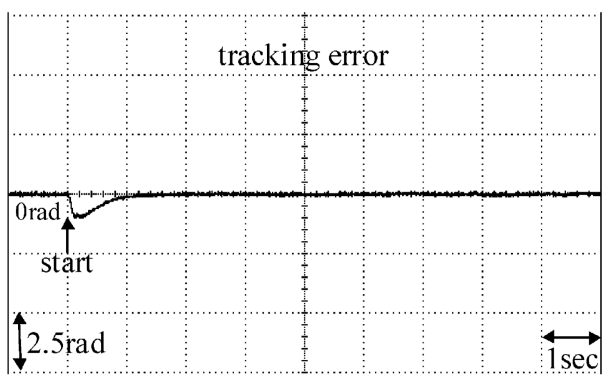

(c)

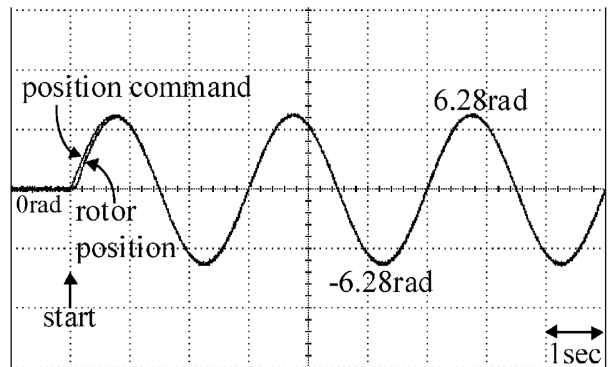

(d)

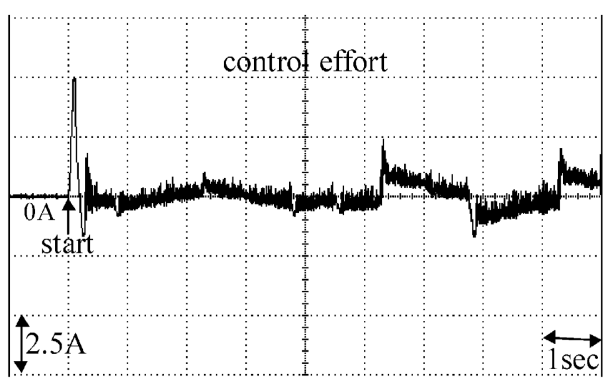

(e)

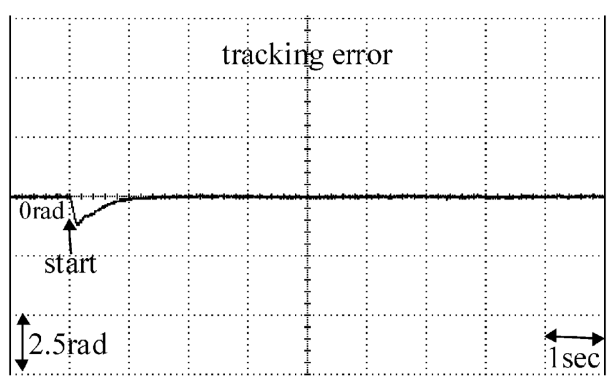

(f)

Fig. 9. Experimental results of AFSMC system with bound estimation for periodic sinusoidal command: (a) position response at external disturbance condition; (b) control effort at external disturbance condition; (c) tracking error at external disturbance condition; (d) position response at parameter variation condition; (e) control effort at parameter variation condition, (f) tracking error at parameter variation condition.

with bound estimation, not only favorable tracking responses can be ensured but also the chattering phenomenon can be much reduced by the estimation mechanism. Two main contributions of this paper are (1) automatic design of fuzzy controller based on stability theory, i.e. the design of such controller is surely stable; and (2) using sliding-mode control technique enables simpler rule base. A comparison of the control characteristics of the FSMC, AFSMC, and AFSMC with bound estimation is summarized in Table 2. From Table 2, the AFSMC with bound estimation design method yields superior control performance without chattering phenomena than other control schemes. 


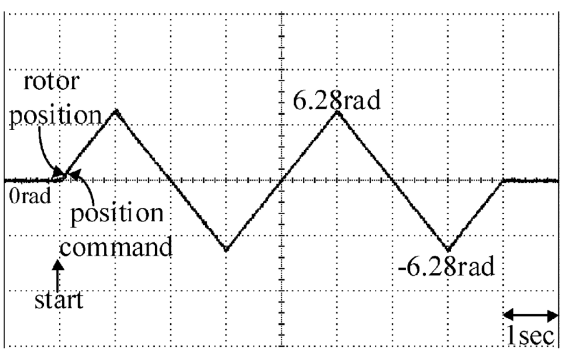

(a)

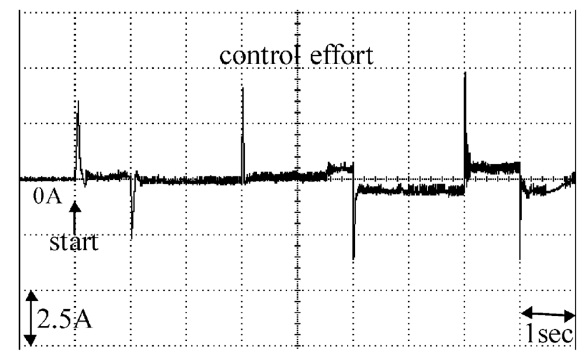

(b)

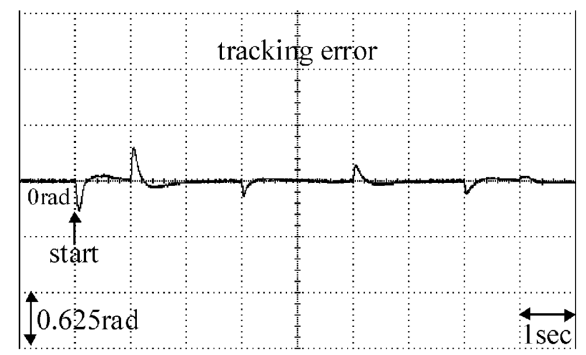

(c)

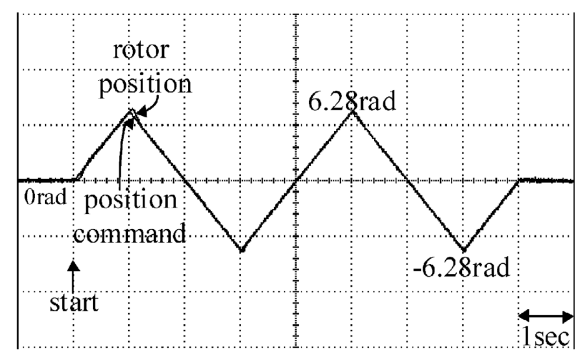

(d)

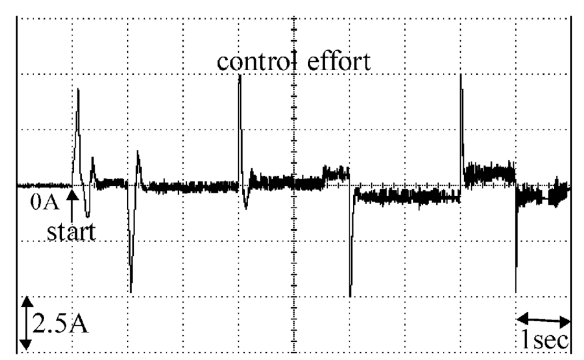

(e)

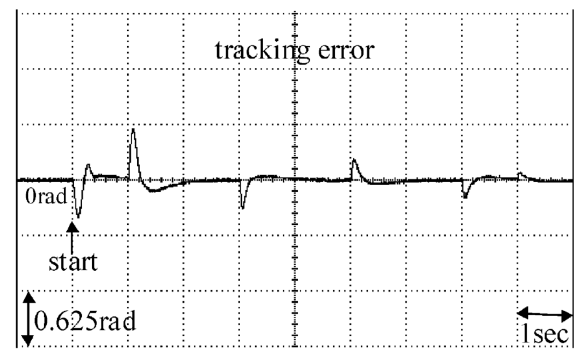

(f)

Fig. 10. Experimental results of AFSMC system with bound estimation for periodic triangular command: (a) position response at external disturbance condition; (b) control effort at external disturbance condition; (c) tracking error at external disturbance condition; (d) position response at parameter variation condition; (e) control effort at parameter variation condition, (f) tracking error at parameter variation condition.

Table 2

Comparison of FSMC, AFSMC and AFSMC with bound estimation

\begin{tabular}{lllll}
\hline Control system & $\begin{array}{l}\text { Fuzzy } \\
\text { rule base }\end{array}$ & $\begin{array}{l}\text { Robust } \\
\text { characteristic }\end{array}$ & $\begin{array}{l}\text { Control } \\
\text { effort }\end{array}$ & $\begin{array}{l}\text { Chattering } \\
\text { phenomenon }\end{array}$ \\
\hline $\begin{array}{l}\text { Fuzzy sliding mode } \\
\text { control system }\end{array}$ & Trial-and error & Degenerate & Medium & None \\
$\begin{array}{l}\text { Adaptive fuzzy sliding } \\
\text { mode control system }\end{array}$ & On-line learning & Good & Medium & Large \\
$\begin{array}{l}\text { Adaptive fuzzy sliding mode control } \\
\text { system with bound estimation }\end{array}$ & On-line learning & Good & Medium & Small \\
\hline
\end{tabular}




\section{Acknowledgements}

The authors would like to acknowledge the financial support of the National Science Council in Taiwan, ROC through its grant NSC 90-2213-E-155-003. Moreover, the authors would like to express their gratitude to the Referees and the Editors-in-Chief for their useful comments and suggestions.

\section{References}

[1] B.K. Bose, Power Electronics and AC Drives, Prentice-Hall, Englewood Cliffs, NJ, 1986.

[2] B.J. Choi, S.W. Kwak, B.K. Kim, Design of a single-input fuzzy logic controller and its properties, Fuzzy Sets and Systems 106 (1999) 299-308.

[3] Y.T. Kim, Z. Bien, Robust self-learning fuzzy controller design for a class of nonlinear MIMO systems, Fuzzy Sets and Systems 111 (2000) 117-135.

[4] C.C. Lee, Fuzzy logic in control systems: fuzzy logic controller-Part I/II, IEEE Trans. Systems Man. Cybernet 20 (1990) 404-435.

[5] C.M. Liaw, F.J. Lin, Position control with fuzzy adaptation for induction servomotor drive, IEE Proc. Electr. Power Appl. 142 (1995) 397-404.

[6] F.J. Lin, S.L. Chiu, Adaptive fuzzy sliding-mode control for PM synchronous motor drives, IEE Proc. Electr. Power Appl. 145 (1998) 63-72.

[7] F.J. Lin, H.M. Su, H.P. Chen, Induction motor servo drive with adaptive rotor time-constant estimation, IEEE Trans. Aerospace Electron. System 34 (1998) 224-234.

[8] R. Palm, Robust control by fuzzy sliding mode, Automatica 9 (1994) 1429-1437.

[9] J.J.E. Slotine, W. Li, Applied Nonlinear Control, Prentice-Hall, Englewood Cliffs, NJ, 1991.

[10] J.R. Timothy, Fuzzy Logic with Engineering Application, McGraw-Hill, New York, 1995.

[11] D.L. Tsay, H.Y. Chung, C.J. Lee, The adaptive control of nonlinear systems using the sugeno-type of fuzzy logic, IEEE Trans. Fuzzy Systems 17 (1999) 225-229.

[12] Y.Y. Tzou, S.Y. Lin, Fuzzy-tuning current-vector control of a three phase PWM inverter for high-performance AC drives, IEEE Trans. Ind. Electron 45 (1998) 782-791.

[13] L.X. Wang, Adaptive Fuzzy Systems and Control: Design and Stability Analysis, Prentice-Hall, Englewood Cliffs, NJ, 1994.

[14] C.C. Wong, J.Y. Chen, Fuzzy control of nonlinear systems using rule adjustment, IEE Proc. Control Theory Appl. 146 (1999) 578-584.

[15] X. Yu, Z. Man, B. Wu, Design of fuzzy sliding-mode control systems, Fuzzy Sets and Systems 95 (1998) $295-306$. 